\title{
Mechanism for the failure of the Edwards hypothesis in the Sherrington-Kirkpatrick spin glass
}

\author{
P. R. Eastham, ${ }^{1}$ R. A. Blythe, ${ }^{2}$ A. J. Bray, ${ }^{3}$ and M. A. Moore ${ }^{3}$ \\ ${ }^{1}$ Cavendish Laboratory, Madingley Road, Cambridge, CB3 OHE, United Kingdom \\ ${ }^{2}$ School of Physics, University of Edinburgh, Edinburgh EH9 3JZ, United Kingdom \\ ${ }^{3}$ School of Physics and Astronomy, University of Manchester, Manchester, M13 9PL, United Kingdom
}

(Received 30 June 2006; published 28 July 2006)

\begin{abstract}
The dynamics of the Sherrington-Kirkpatrick model at $T=0$ starting from random spin configurations is considered. The metastable states reached by such dynamics are atypical of such states as a whole, in that the probability density of site energies, $p(\lambda)$, is small at $\lambda=0$. Since virtually all metastable states have a much larger $p(0)$, this behavior demonstrates a qualitative failure of the Edwards hypothesis. We look for its origins by modeling the changes in the site energies during the dynamics as a Markov process. We show how the small $p(0)$ arises from features of the Markov process that have a clear physical basis in the spin glass, and hence explain the failure of the Edwards hypothesis.
\end{abstract}

DOI: 10.1103/PhysRevB.74.020406

PACS number(s): 75.10.Nr, 45.70.-n, 75.50.Lk, 81.05.Rm

Complex systems like granular media have a large number of metastable (blocked) configurations. When shaken or tapped, they quickly relax into another metastable state. A subsequent tap will result in another blocked or jammed state, and so on. The complexity (entropy) of metastable states in granular systems or spin glasses is extensive in the system size. Edwards and co-workers have proposed that the quasiequilibrium steady state which results from repeated tapping can be described using a thermodynamic measure over the metastable states. ${ }^{1,2}$ The strongest version of such a hypothesis predicts that a system adopts configurations that maximize the entropy. In weaker versions parameters such as the energy or volume are fixed, and the system adopts configurations that maximize the entropy consistent with the constraints.

Edwards hypotheses have met with a high degree of success in many complex systems. Some recent examples include predicting (i) the distribution of contact forces, ${ }^{3}$ and the effective temperature, ${ }^{4}$ in simulations of granular media, (ii) the dynamical entropy and correlation functions in the slow-dynamics regime of the Kob-Anderson model, ${ }^{5}$ and (iii) the distribution of steady-state energies in the tapped Sherrington-Kirkpatrick model. ${ }^{6}$ They give some good approximations for the zero-temperature constrained dynamics of finite-dimensional Ising ferromagnets though are known to fail in some respects..$^{7,8}$ We note also support in the context of the slow dynamics of mean-field spin-glass models, where it has been argued that the effective temperature coincides with the Edwards temperature. ${ }^{5,7}$ The underlying general idea that dynamics does not strongly select among metastable states is yet more widely used to attribute slow dynamics to a proliferation of metastable states-in optimization algorithms, ${ }^{9}$ for example.

Here we study dynamics in the the canonical SherringtonKirkpatrick (SK) model, for which the metastable states are already well understood. ${ }^{10,11}$ We show that the metastable states selected by dynamics are of a very special character in which the energy $2 \lambda_{i}$ to flip the spin at site $i$ has a distribution $p(\lambda)$ which is small for $\lambda \approx 0$. Generic metastable states have $p(0) \neq 0$. The dynamically selected metastable states are a vanishing fraction of the totality of metastable states in the thermodynamic limit and therefore, according to the Edwards hypothesis, should not be expected to be selected. We provide a model of the dynamics that explains why it converges onto this tiny subset of the metastable states.

The SK Hamiltonian is $H=-\Sigma_{(i j)} J_{i j} S_{i} S_{j}=-\frac{1}{2} \Sigma_{i} \lambda_{i}$, where $S_{i}= \pm 1, \lambda_{i}=S_{i} \sum_{j \neq i} J_{i j} S_{j}$ is the "site energy," equal to one-half of the energy change on flipping the spin $S_{i}$, and $\Sigma_{(i j)}$ indicates a sum over all pairs of sites. The interaction strengths $J_{i j}$ are independent random variables from a Gaussian distribution with zero mean and standard deviation $1 / \sqrt{N}$.

We consider the nonequilibrium behavior of the model under single-spin relaxational dynamics, ${ }^{12-14}$ starting from a random initial state. We consider the $T=0$ limit of this dynamics, as in Refs. 6, 7, and 15, because it allows the metastable states to be clearly identified. Further motivation for studying this limit comes from its use in contexts ranging from hysteresis in the SK model ${ }^{16}$ to domain growth in ferromagnets; it corresponds to the basic Hopfield neuralnetwork algorithm, and to the greedy steps in the WALK-SAT algorithm. ${ }^{9}$

The state evolves by flipping single spins with $\lambda_{i}<0$, i.e., those that are opposed to the local magnetic field on their site, until no such spins remain. Different choices for the order of spin flips lead to different versions of the algorithm. In the "sequential" algorithm a randomly selected unstable spin is flipped at each timestep, while in the "greedy" algorithm the most unstable (minimum $\lambda_{i}$ ) spin is flipped. The behavior of these different algorithms is remarkably similar. ${ }^{15}$

The $T=0$ dynamics of the SK model converges onto onespin-flip-stable states, in which every spin aligns with its local field. This model is an attractive one in which to consider the Edwards hypothesis, because these metastable states have been studied analytically. ${ }^{10,11}$ The key results are shown in Fig. 1, in which the calculated and measured entropy ("complexity") and distribution of local energies of the metastable states are compared. The converged energies do not cluster at the peak of the complexity curve, but are instead clustered in a narrow range around $E \approx-0.7$, so the dynamics certainly does not sample the metastable states uniformly. Furthermore, the computed $p(\lambda)$ is qualitatively 

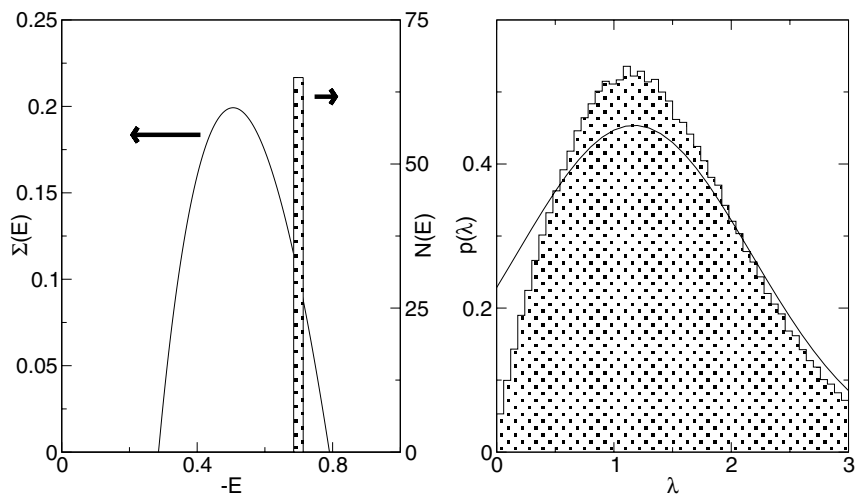

FIG. 1. Discrepancies between simulations of sequential spinglass dynamics on a system of 5000 spins and the analytical predictions based on flat-measure assumptions. Left panel, curve (left axis): Complexity of metastable states of energy $E, \Sigma(E)$ $=(1 / N) \ln N_{s}(E)$ for the SK model, where $N_{s}(E)$ is the mean number of metastable states with energy $E$. Bar (right axis): Histogram of the converged energies for 65 runs of the sequential spin-glass dynamics. Right panel, curve: Average $p(\lambda)$ in the metastable states of energy -0.7 . Histogram: Average $p(\lambda)$ over the final states of the spin-glass dynamics.

different from the flat-average $p(\lambda)$ at the converged energy: The computed $p(\lambda)$ has a negligible intercept, whereas the flat-average $p(\lambda)$ has a significant finite intercept. Thus the dynamics does not uniformly sample the metastable states at the converged energy. Furthermore, repeated "tapping" of a randomly selected fraction of spins does not alter this conclusion: In our simulations the steady state does not develop an intercept. Thus the states reached by the dynamics are always qualitatively different from the totality of metastable states of the same energy, and in the thermodynamic limit they are a negligible fraction of these states. In other situations, it has been observed that the blocked states reached by the dynamics have different energies to those typical of the blocked states as a whole. ${ }^{17}$ Our work shows this feature too, but furthermore that the dynamically generated states are even atypical of the states of the same energy.

To understand why the typical metastable states are not realized we must look to the dynamics. We simplify the problem by considering only the population of site energies $\{\lambda\}$ and making the working assumption that the evolution of $p(\lambda)$ can be modeled in terms of a Markov process in this population.

The population dynamics is designed to parallel the real spin-glass dynamics. At each step an unstable spin $i$ is flipped, corresponding to $\lambda_{i} \rightarrow-\lambda_{i}$. In the spin glass the other site energies $\lambda_{j}$ shift by an amount

$$
\Delta \lambda_{j ; i}=-2 S_{i} S_{j} J_{i j}
$$

Here $S_{i}$ and $S_{j}$ denote the spin configuration before the flip. To obtain a population dynamics we replace the drifts $\Delta \lambda_{j ; i}$ with functions of the site energies. In the Markov approximation we replace them with independent random variables, whose distribution $P\left(\Delta \lambda_{j ; i} \mid\{\lambda\}\right)$ depends only upon the site energies at each step.
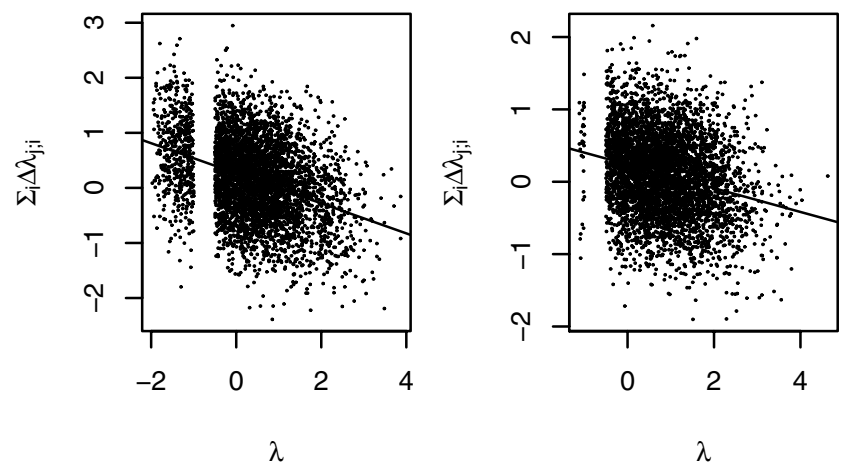

FIG. 2. Total changes in the site energies, $\Sigma_{i} \Delta \lambda_{j ; i}$ of unflipped spins when all spins with site energies in the ranges $-1.0<\lambda_{i}$ $<-0.5$ are flipped, in configurations generated by 100 (left panel, 671 flipped spins), and 500 (right panel, 420 flipped spins) steps of the greedy algorithm on a system of 5000 spins. This algorithm converged after 2465 flips. The straight lines show linear fits to the data.

Similar approaches have previously been applied to the SK model, ${ }^{13,18}$ granular media, ${ }^{1}$ the WALK-SAT algorithm, ${ }^{9}$ and spin models on random graphs. ${ }^{14,17}$ Previous work on the SK model has attempted to calculate $P\left(\Delta \lambda_{j ; i} \mid\{\lambda\}\right)$. Although this approach has met with some success, ${ }^{18}$ it leads to very involved models. Owing to their complexity, these models are only tractable numerically, and their physics remains obscure. We therefore take a different approach, which is to determine the general features of $P\left(\Delta \lambda_{j ; i} \mid\{\lambda\}\right)$ that suffice for a qualitative understanding of the dynamics.

We can deduce some of the general features of $P\left(\Delta \lambda_{j ; i} \mid\{\lambda\}\right)$ directly from (1). Because the model is completely connected, summing the drifts over all the unflipped spins gives the sum rule $\Sigma_{j \neq i} \Delta \lambda_{j ; i}=-2 \lambda_{i}$. Therefore, to model the dynamics with a Markov process, we must take $P\left(\Delta \lambda_{j ; i} \mid\{\lambda\}\right)$ to have a mean proportional to $1 / N$ in the large$N$ limit. Since $S^{2}=1$, the variance of the drifts is then just associated with that of the bond distribution,

$$
\left\langle\Delta \lambda_{j ; i}^{2}\right\rangle-\left\langle\Delta \lambda_{j ; i}\right\rangle^{2} \sim 4 / N
$$

Our simulations of the spin-glass dynamics converged in $\leq N$ flips. Since $J_{i j}=O(1 / \sqrt{N})$, the third and higher cumulants of $\Delta \lambda_{j ; i}$ are higher order in $1 / N$ than the mean and variance. Therefore the total drift produced by the higher cumulants is negligible over the convergence time, and we may take $P\left(\Delta \lambda_{j ; i} \mid\{\lambda\}\right)$ to be Gaussian. Any correlations between the drifts $\Delta \lambda_{j ; i}$ and the fields $\lambda_{j}$ would have a qualitative effect on the evolution of $p(\lambda)$. We looked for such correlations by taking the states generated during the spin-glass dynamics and numerically evaluating the drifts when spins are flipped. The results are shown in Fig. 2. Each point is the total drift of an unflipped spin as a function of its site energy when all spins with site energies in a small range are flipped.

Note the general correlation between the drifts and the site energies, which can be seen in Fig. 2. The overall drift on flipping a spin $i$ is fixed by the sum rule but it is nonuniformly distributed among spins according to their energies: 
Highly unstable spins tend to have their site energies strongly increased, at the expense of a reduced increase or a decrease in the site energies of the more stable spins. This is physically reasonable because a very unstable spin has mostly unsatisfied bonds, while a very stable spin has mostly satisfied bonds. Therefore the spin $i$ is likely to be connected to a highly unstable spin by an unsatisfied bond, and to a highly stable spin by a satisfied bond, producing the observed correlation.

We now consider whether the general features we have identified can explain aspects of the spin-glass dynamics, in particular the observation that it apparently converges in $\sim N$ time steps, to a state with a small intercept and an approximately linear $p(\lambda)$. We adopt the following minimal model, which captures the behavior of the distribution $p(\lambda)$ at small $\lambda$ and at late times. We make the simplest assumption, that the drift $\Delta \lambda_{j ; i}$ in the value of $\lambda_{j}$ resulting from flipping an unstable spin $i$ is a Gaussian random variable with mean $c / N$ $(c>0)$ and variance $\sigma^{2} / N$, where, according to Eq. (2), $\sigma^{2}$ $=4$. This assumption is motivated by the correlations visible in Fig. 2, which lead us to expect that the mean drift of a low-energy spin is nonvanishing as the converged state is approached. Since the assumption of a constant drift violates the previously derived sum rule, it cannot be correct for all sites. Our model is designed to address the behavior of $p(\lambda, t)$ at small $\lambda$.

The equation of motion for $p(\lambda, t)$ is, for large $N$,

$$
\begin{aligned}
\frac{\partial p(\lambda, t)}{\partial t}= & \frac{1}{q(t)}[p(-\lambda, t) \theta(\lambda)-p(\lambda, t) \theta(-\lambda)] \\
& -c \frac{\partial p(\lambda, t)}{\partial \lambda}+\frac{\sigma^{2}}{2} \frac{\partial^{2} p(\lambda, t)}{\partial \lambda^{2}}
\end{aligned}
$$

where $q(t)=\int_{-\infty}^{0} p(\lambda, t) d \lambda$ is the weight in the negative side of the distribution (from which the flipped spins are drawn) at time $t$, and the units of time are such that there are $N$ moves per unit time.

The first term in (3) derives from the flipping process $\lambda_{i}$ $\rightarrow-\lambda_{i}$, which simply transfers the population from negative to positive $\lambda$ at a rate of 1 spin per time step. The second term derives from the mean of the drifts, which leads, within our model, to a uniform convection in the $\lambda$ space. The final diffusion term is due to the fluctuations in the drifts. All these processes occur on the same time scale, taking $\sim N$ steps, or a time $\sim N^{0}$, to produce an effect of order 1 on $p(\lambda, t)$.

To understand the solutions to Eq. (3) we first consider the case $\sigma^{2}=0$. The equation of motion can then be solved analytically, to give $p(\lambda, t)$ in terms of integrals over $p(\lambda, 0)$. The results are shown in the top panel of Fig. 3, for a Gaussian initial condition and $c=4$. The number of spins with $\lambda$ $<0$ is always decreasing at a finite rate, due to the convection across $\lambda=0$ and the flipping process. Thus this process certainly converges, reaching $q(t)=0$ in a finite time. In general the decay of $p(0, t)$ near the end of the evolution is linear in time, which combines with the convection to produce a linear $p(\lambda)$, with no intercept, in the converged state. The slope depends on the initial conditions and on $c$. It diverges
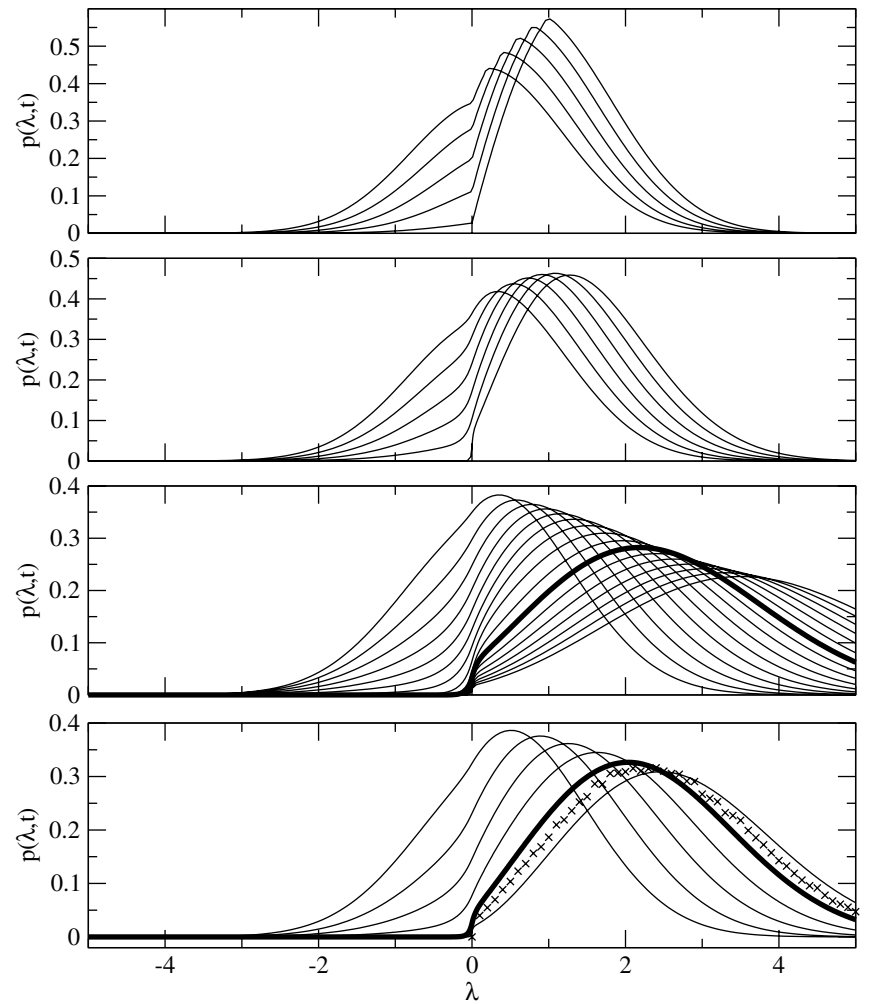

FIG. 3. The solution to (3), with a Gaussian initial condition and $c=4$ (top three panels) and 8 (lowest panel), for $\sigma^{2}=0$ (top panel), 1 (middle panel), and 4 (lower two panels). Curves are plotted at time intervals of 0.05 . The bold curves in the lower two figures are the earliest at which $p(0, t) \approx 0.03$. They agree with the histograms obtained by direct simulation of the population dynamics model with 5000 fields, shown for $c=8$ (crosses).

as $c \rightarrow 0$, where the resulting $p(\lambda)$ is just the half Gaussian created by the flipping.

For $\sigma^{2}>0$, we have solved (3) numerically. The resulting $p(\lambda, t)$ are shown in the lower three panels of Fig. 3. For these values of $c$ and $\sigma^{2}$ the behavior at early times is similar to that with $\sigma^{2}=0$. The diffusion, however, smooths out the singularities (discontinuity of slope at $\lambda=0$ ) evident in the $\sigma^{2}=0$ solutions, and broadens the distribution, but the tail of unstable spins continues to decay at a significant rate. This can be understood by noting that while the positive slope at $\lambda=0$ leads to a diffusion current back toward $\lambda<0$, for these parameters this current is too small to overcome the loss due to flipping and convection. In contrast, if $c$ is too small the solution with $\sigma=0$ would have a large average slope at $\lambda$ $=0$, and the diffusion would have a major effect.

Although for some $c$ and $\sigma^{2}$ the early-time behavior is similar to that of the model with $\sigma^{2}=0$, we see that a new regime appears at later times. As the tail of unstable spins becomes narrower, the slope at $\lambda=0$ increases, while the intercept continues to decay. This slows the decay of $q(t)$, which obeys $d q / d t=-1-c p(0, t)+\left.\left(\sigma^{2} / 2\right)(\partial p / \partial \lambda)\right|_{\lambda=0}$, with terms due to flipping, convection, and diffusion, respectively. Indeed, in the the lower two panels the slope at $\lambda=0$ is approaching the critical slope of $2 / \sigma^{2}$ at which the diffusion current balances the loss due to flipping. $q(t)$ must continue 
to decay, since the bulk of $p(\lambda)$ will continue to diffuse and convect, and by continuity this must reduce the tail of unstable spins. However this decay is extremely slow. Furthermore, it is an artifact of our low-energy approximation, in which we replaced the $\lambda$-dependent convection rate by a constant. In a more complete treatment the biasing visible in Fig. 2 would tend to confine the bulk of the distribution to a region centered on $\lambda \sim 1$, due to negative convection rates at large $\lambda$, whereas in the model the maximum of the distribution continues to drift to the right-see Fig. 3. We note that the approximation scheme of Ref. 18 gives such negative convection rates at large $\lambda .{ }^{19}$

In an infinite system the Markov process and the spinglass dynamics terminate when $q(t)=0$. Our numerics suggest that this does not occur in a finite time for the Markov process, unless $\sigma=0$. Hence it is inconsistent with the conjecture that the dynamics of the infinite spin glass converges in a finite time. However, for moderate values of $c$ the features in $p(\lambda)$ associated with the slowing of the dynamics become so small that it would require a very large system for them to be resolved. Therefore we suggest that the minimal Markov model may be adequate to understand the convergence seen in the spin-glass simulations, which are finite, albeit large.

In the finite spin glass the converged $p(\lambda)$ has a small intercept, which we can estimate by fitting to histograms such as those shown in Fig. 1. For $N=1000$ we obtain an intercept of 0.06 , and $\approx 0.03$ for $N=5000$ and 10000 , consistent with the intercept of $2 / \sqrt{N}$ suggested in Ref. 15 . This scaling is explained by the Markov model, since for $p(0)$ $\lesssim 1 / \sqrt{N}$ the average diffusion flux from positive to negative $\lambda$ is less than the one spin per time step transferred in the opposite direction by the flipping. The dynamics will rapidly converge after such an intercept is reached, with little further change in $p(\lambda)$.

Based on these arguments and the results for the direct simulations of the spin glass, we suggest that the Markov process will converge in a finite system when $p(0, t)$ obtained from Eq. (3) becomes comparable with $1 / \sqrt{N}$. For a large enough $c$, this condition is met before the dynamics becomes dominated by diffusion, and the resulting $p(\lambda, t)$ has some features similar to that of the simulational result. This can be seen in the lower two panels of Fig. 3, where we mark in bold the $p(\lambda, t)$ at which $p(0, t) \approx 0.03$. This corresponds to the smallest intercept we have seen in the spinglass simulations. Direct simulations of the minimal model in a finite population lead to similar distributions.

To conclude, we have discovered a correlation between the energy shifts and site energies in the spin-glass dynamics, and shown that such a correlation can be sufficient for the dynamics to converge to a metastable state in a large but finite system. Since in the population-dynamics approach the converged state will have a nearly continous $p(\lambda)$, while the typical metastable states have a discontinous one, the success of a population-dynamics approach implies the failure of the flat-measure one. Such success is only possible because the population-dynamics converges: otherwise spins would flip many times, and the Markov approximation would fail.

These considerations suggest an unusual picture of the origins of slow dynamics in some complex systems. Disorder and frustration do play a role, captured by the diffusion term, in preventing a fast convergence of the dynamics, but this role is limited by the drift. This causes the dynamics to converge long before it has time to thoroughly explore the state space, and so the Edwards hypothesis fails.

The Edwards hypothesis was shown to correctly predict the form of the distribution of steady-state energies in simulations of tapping the SK model in Ref. 6. Given our results, this agreement now poses an intriguing problem. Perhaps the true dynamical entropy $\Sigma_{d y n}(E)$ has an energy dependence similar to that of the flat-measure entropy $\Sigma_{e d w}(E)$, so that the energy distributions in tapping take similar forms. Since the states are very different, however, it is unclear why this should occur.

We thank David Dean and David Sherrington for useful discussions. P.R.E. acknowledges support from Sidney Sussex College, Cambridge and the Aspen Center for Physics, and R.A.B. from the Royal Society of Edinburgh.
${ }^{1}$ S. F. Edwards, in Granular Media: An Interdisciplinary Approach, edited by A. Mehta (Springer-Verlag, New York, 1994).

${ }^{2}$ S. F. Edwards and A. Mehta, J. Phys. (Paris) 50, 2489 (1989).

${ }^{3}$ P. T. Metzger and C. M. Donahue, Phys. Rev. Lett. 94, 148001 (2005).

${ }^{4}$ H. Makse and J. Kurchan, Nature (London) 415, 614 (2002).

${ }^{5}$ A. Barrat, J. Kurchan, V. Loreto, and M. Sellitto, Phys. Rev. Lett. 85, 5034 (2000).

${ }^{6}$ D. S. Dean and A. Lefèvre, Phys. Rev. Lett. 90, 198301 (2003).

${ }^{7}$ C. Godrèche and J. M. Luck, J. Phys.: Condens. Matter 17, S2573 (2005).

${ }^{8}$ F. Camia, Eur. Phys. J. B 47, 239 (2005).

${ }^{9}$ W. Barthel, A. K. Hartmann, and M. Weigt, Phys. Rev. E 67, 066104 (2003).
${ }^{10}$ A. J. Bray and M. A. Moore, J. Phys. C 13, L469 (1980).

${ }^{11}$ F. Tanaka and S. F. Edwards, J. Phys. F: Met. Phys. 10, 2769 (1980).

${ }^{12}$ R. Glauber, J. Math. Phys. 4, 294 (1963).

${ }^{13}$ S. N. Laughton, A. C. C. Coolen, and D. Sherrington, J. Phys. A 29, 763 (1996).

${ }^{14}$ G. Semerjian and M. Weigt, J. Phys. A 37, 5525 (2004).

${ }^{15}$ G. Parisi, Fractals 11 (Suppl.), 161 (2003).

${ }^{16}$ F. Pázmándi, G. Zaránd, and G. T. Zimányi, Phys. Rev. Lett. 83, 1034 (1999).

${ }^{17}$ J. Berg and A. Mehta, Europhys. Lett. 56, 784 (2001).

${ }^{18}$ H. Horner, Z. Phys. B: Condens. Matter 78, 27 (1990).

${ }^{19} \mathrm{H}$. Horner (private communication). 